Revue des patrimoines

13 | 2010

Art rupestre : la 3D un outil de médiation du réel invisible?

\title{
Relevé 3D de gravures fines paléolithiques dans l'abri du Colombier (gorges de l'Ardèche)
}

Marc Azéma, Bernard Gély, Françoise Prudhomme et ATM 3D Société ATM3D

\section{(2) OpenEdition}

Journals

Édition électronique

URL : http://journals.openedition.org/insitu/6723

DOI : $10.4000 /$ insitu. 6723

ISSN : 1630-7305

Éditeur

Ministère de la Culture

Référence électronique

Marc Azéma, Bernard Gély, Françoise Prudhomme et ATM 3D Société ATM3D, « Relevé 3D de gravures fines paléolithiques dans l'abri du Colombier (gorges de l'Ardèche) », In Situ [En ligne], 13 | 2010, mis en ligne le 16 avril 2012, consulté le 14 novembre 2019. URL : http:// journals.openedition.org/insitu/6723 ; DOI : 10.4000/insitu.6723

Ce document a été généré automatiquement le 14 novembre 2019.

\section{(†) $\odot$

In Situ Revues des patrimoines est mis à disposition selon les termes de la licence Creative Commons Attribution - Pas d'Utilisation Commerciale - Pas de Modification 4.0 International. 


\section{Relevé 3D de gravures fines paléolithiques dans l'abri du Colombier (gorges de l'Ardèche)}

Marc Azéma, Bernard Gély, Françoise Prudhomme et ATM 3D Société ATM3D

Figure 1

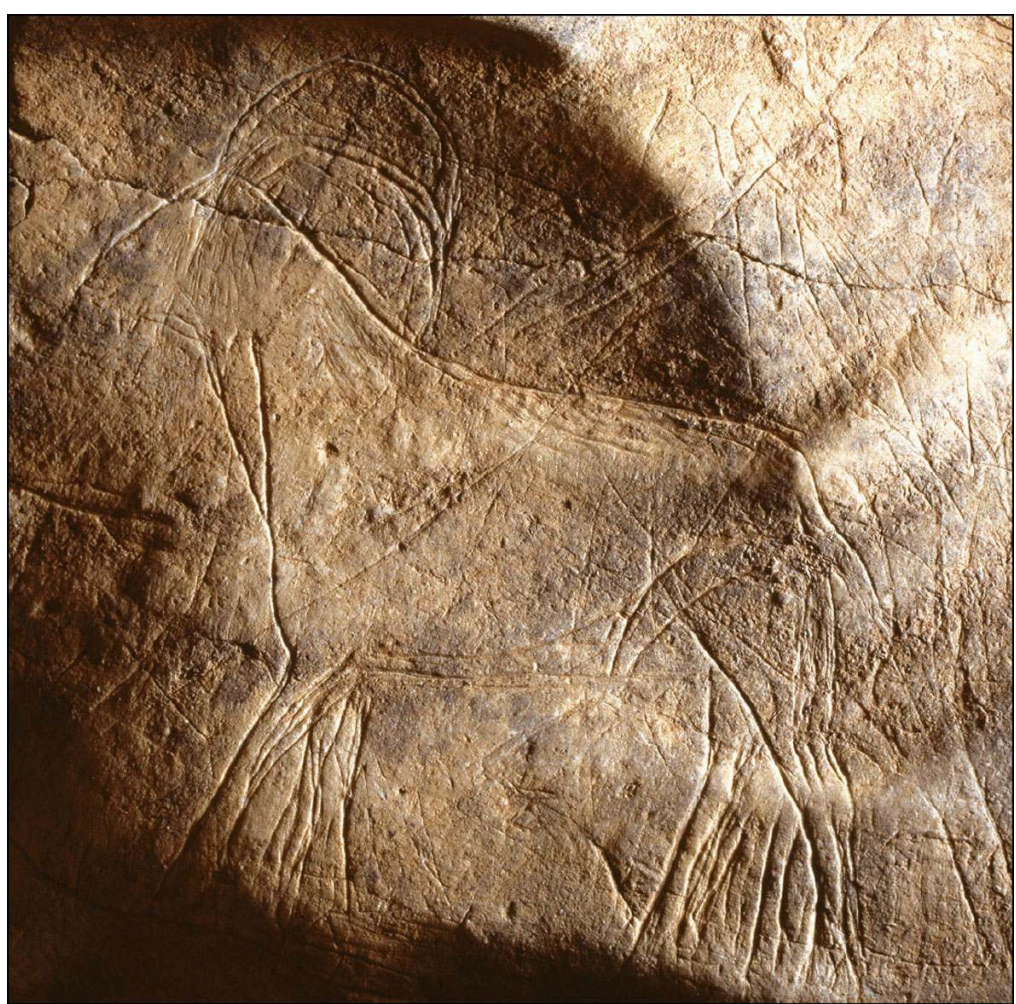

Le bouquetin à pattes multiples (« en marche ») de l'abri du Colombier (Ardèche)

Phot. Norbert Aujoulat. (c) Ministère de la Culture, DAPA, CNP, avec l'autorisation du propriétaire, M. Henry Helly 
1 L'exposition temporaire "Préhistoire de la bande dessinée et du dessin animé " ${ }^{1}$ consacrée à la vulgarisation d'une partie des travaux de Marc Azéma sur la représentation du mouvement dans l'art préhistorique ${ }^{2}$ a été l'occasion de réaliser une opération de relevé 3D (scan laser) sur un abri gravé magdalénien dont les tracés, d'une exceptionnelle finesse, sont soumis aux aléas naturels et humains. Les objectifs de cette opération relevaient donc de l'étude, de la conservation et de la valorisation d'un art pariétal susceptible d'altérations relativement rapides (fig. $\left.\mathbf{n}^{\circ} \mathbf{1}\right)$.

\section{L'exposition}

2 Co-produite par le musée régional de Préhistoire d'Orgnac (Ardèche) ${ }^{3}$, le Centre de préhistoire du Pech-Merle de Cabrerets (Lot) et la société de production Passé Simple ${ }^{4}$, l'exposition a pour objectif de présenter au public la genèse et l'évolution d'un médium universel : la narration graphique et sa forme actuelle, la «bande dessinée » (BD). Elle s'appuie en grande partie sur les recherches récentes du préhistorien et réalisateur Marc Azéma, spécialisé dans les nouvelles technologies, qui tendent à démontrer scientifiquement que les origines de la figuration narrative (BD) remontent à la Préhistoire ${ }^{5} 68$ tout comme celles de l'animation séquentielle ou « dessin animé ».

3 Ainsi, la bande dessinée et le dessin animé, considérés comme des formes relativement récentes d'art populaire, se voient valorisées par leurs origines préhistoriques. Les nombreux exemples puisés dans l'histoire de l'art démontrent que l'Homme a depuis toujours pratiqué ces processus narratifs, qui permirent un jour de passer du mythogramme au pictogramme puis à l'écriture.

L'exposition présente de nombreux exemples d'art séquentiel provenant de plusieurs cavités (Chauvet, Trois-Frères) et de toutes les périodes du Paléolithique supérieur. Certaines images sont de véritables petits dessins animés figés sur la pierre comme le bouquetin de l'abri du Colombier dans les gorges de l'Ardèche (voir fig. $\mathrm{n}^{\circ} 1$ ) : l'animal est représenté en train de trottiner, bêlant tête haute, entouré des autres congénères constituant une petite harde. Le mouvement rapide des membres est signifié par une multiplication des contours générant ainsi une sorte de flou graphique $e^{91011} 12$.

5 Afin de compléter cette présentation muséographique par des éléments provenant de grottes ardéchoises inaccessibles au public, les concepteurs de l'exposition ont décidé de réaliser un relevé 3D précis par scan au laser du panneau supportant cette œuvre étonnante ( $20 \mathrm{~cm} \times 20 \mathrm{~cm}$ environ).

\section{Présentation du site}

\section{Situation}

6 Le site archéologique du Colombier est situé dans le périmètre protégé de la Réserve nationale naturelle des gorges de l'Ardèche, sur la rive droite et au pied des falaises du Saleyron, à une trentaine de mètres au-dessus de la rivière. 
Figure 2

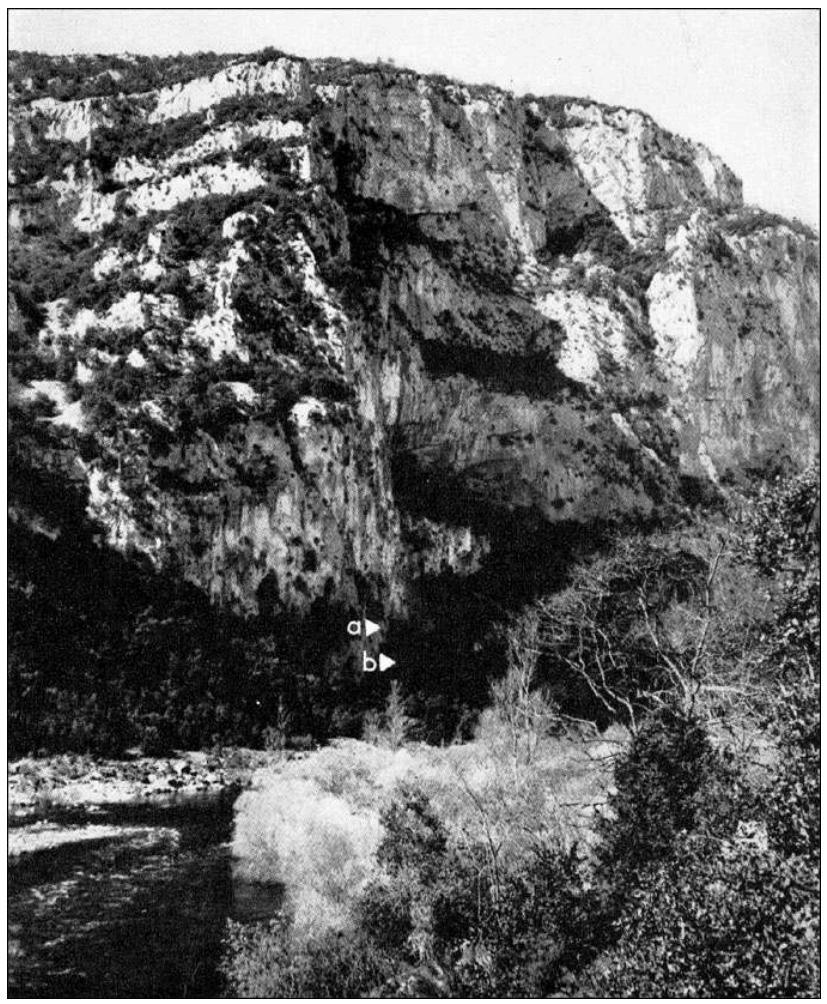

a - Porche de la grotte du Colombier. b - Abris du Colombier

Droits réservés

7 Il comprend trois cavités attenantes et communicantes qui ont livré des vestiges magdaléniens et aziliens (fig. $\mathbf{n}^{\circ}$ 2) ; la grotte, qui atteint $300 \mathrm{~m}$ de longueur environ, est située au-dessus de deux abris-sous-roche qui furent utilisés autrefois comme bergerie. Par commodité, les cavités seront décrites séparément, bien qu'elles fussent à l'origine un seul habitat bien stratifié et richement documenté mais très amoindri par l'érosion et les activités humaines ${ }^{13}$.

\section{Découverte des gravures paléolithiques}

\section{La grotte (Colombier 1)}

8 Signalée dès le $\mathrm{XIX}^{\mathrm{e}}$ siècle, la grotte du Colombier a été reconnue en tant que site préhistorique en 1947 par l'abbé Glory. Il y a authentifié et relevé un ensemble de gravures dont la découverte revient à deux spéléologues, G. Claron et A. Crouzet.

9 À environ cinquante mètres de l'entrée, des figures de grands herbivores (un aurochs, deux bouquetins et un probable cervidé) et des signes (ovales striés, bâtonnets...) sont dispersés sur les parois de l'unique galerie. Très finement tracées, les gravures se distinguent difficilement.

10 À l'entrée de la grotte, des fouilles ont révélé plusieurs séquences d'occupation datant de la fin du Paléolithique supérieur : sous les niveaux riches en pointes aziliennes, les niveaux magdaléniens ont livré une industrie osseuse (sagaies, harpons à double rang de barbelures, aiguilles) comparable à celle retrouvée par la suite dans l'abri. 


\section{L'abri inférieur (Colombier 2)}

11 S'ouvrant quelques mètres en contrebas du porche de la grotte, l'abri du Colombier a fait l'objet d'une fouille archéologique par A. Héritier, à partir de 1956.

Les premiers blocs gravés furent reconnus, à cette occasion, dans la couche inférieure azilienne $n^{\circ} 3$ : «plusieurs blocs de calcaire... d'assez grandes dimensions... gravés en tous sens de traits fins... décombres d'une décoration pariétale antérieure... et, selon $\mathrm{R}$ Desbrosse, l'un d'eux porterait une tête de bouquetin. ${ }^{14}$.

13 De 1973 à 1976, une reprise des travaux par P. Ayrolles a dégagé la base des parois où, au cours d'une visite ultérieure furent perçues, par J.-L. Porte, des gravures en place. Enfin, en 1990, les fouilles de G. Onoratini ont mis au jour, dans les couches archéologiques, des fragments gravés détachés des parois par le gel.

14 Les gravures de l'abri sont similaires à celles de la grotte, par leurs thèmes comme par leur facture. La multiplication des tracés modelant les pattes du bouquetin central apparaît comme une volonté de décomposer le mouvement.

Figure 3

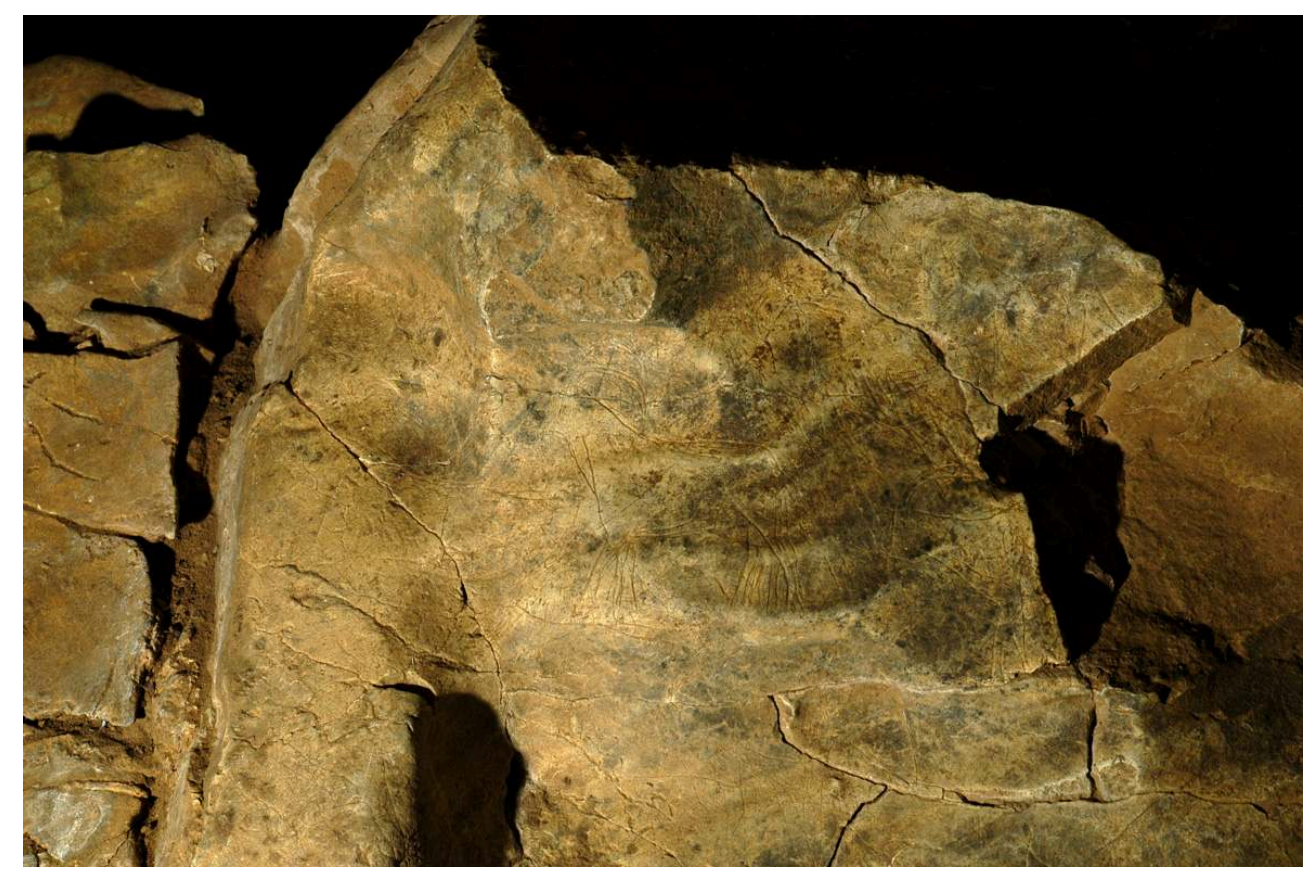

Bloc rocheux supportant le panneau du bouquetin à pattes multiples, abri inférieur du Colombier Phot. Marc Azéma. (c) Marc Azéma, avec l'autorisation du propriétaire, M. Henry Helly

15 L'opération de relevé 3D a porté, plus particulièrement, sur un pan rocheux oblique dont la partie supérieure est entièrement décorée de gravures fines, parfois entremêlées et superposées, où l'on discerne surtout des bouquetins mâles ainsi qu'un petit signe ovale bouclé, analogue à ceux repérés dans la grotte, mais plus petit (fig. $\left.\mathbf{n}^{\circ} 3\right)$.

16 Cet ensemble décoré était recouvert par des cailloutis cendreux équivalents à la couche 13 (Magdalénien terminal) ou par le sommet des cailloutis cryoclastiques de la couche $15 \mathrm{~B}$ qui a livré des écailles de calcaire décorées ${ }^{15}$. Ce recouvrement par des couches archéologiques au mobilier caractéristique de la fin du Magdalénien fait de cet 
ensemble un excellent repère chronologique pour la connaissance de l'art des gorges de l'Ardèche.

17 Le bloc gravé est en bon état de conservation grâce à son enfouissement prolongé. Cependant, depuis leur découverte, les gravures sont à l'air libre et soumises aux aléas naturels et humains : les parois gravées ont, par endroits, subi des dégradations par burinage.

\section{Le scan 3D}

\section{Objectifs}

L'opération de relevé ${ }^{16}$ non vulnérante, avait pour but :

- de tester les techniques de relevé par scan au laser 3D sur des gravures très fines ;

- de conserver une archive de l'état des gravures au moment du relevé 3D ;

- de réaliser un documentaire didactique sur l'opération destiné à la projection dans le cadre de l'exposition;

- de réaliser, à partir des fichiers numériques, une étude scientifique de la technique de gravure.

19 Il faut souligner que malgré la campagne de photos et de moulages (1981), les gravures de l'abri n'ont jamais fait l'objet de véritables relevés.

20 Ainsi, ce projet offrit l'opportunité de favoriser la conjonction d'intérêts d'études scientifiques, de mesures conservatoires et de restitution d'œuvres pariétales inaccessibles au public.

\section{But conservatoire}

21

Les fichiers obtenus sont représentatifs de l'état en 2007 du bloc gravé et permettent de suivre les effets dans le temps des éléments naturels, en visualisant à moyen et long terme l'évolution des altérations. Il faut donc procéder au relevé intégral du bloc et non pas uniquement des gravures.

\section{But scientifique}


Figure 4

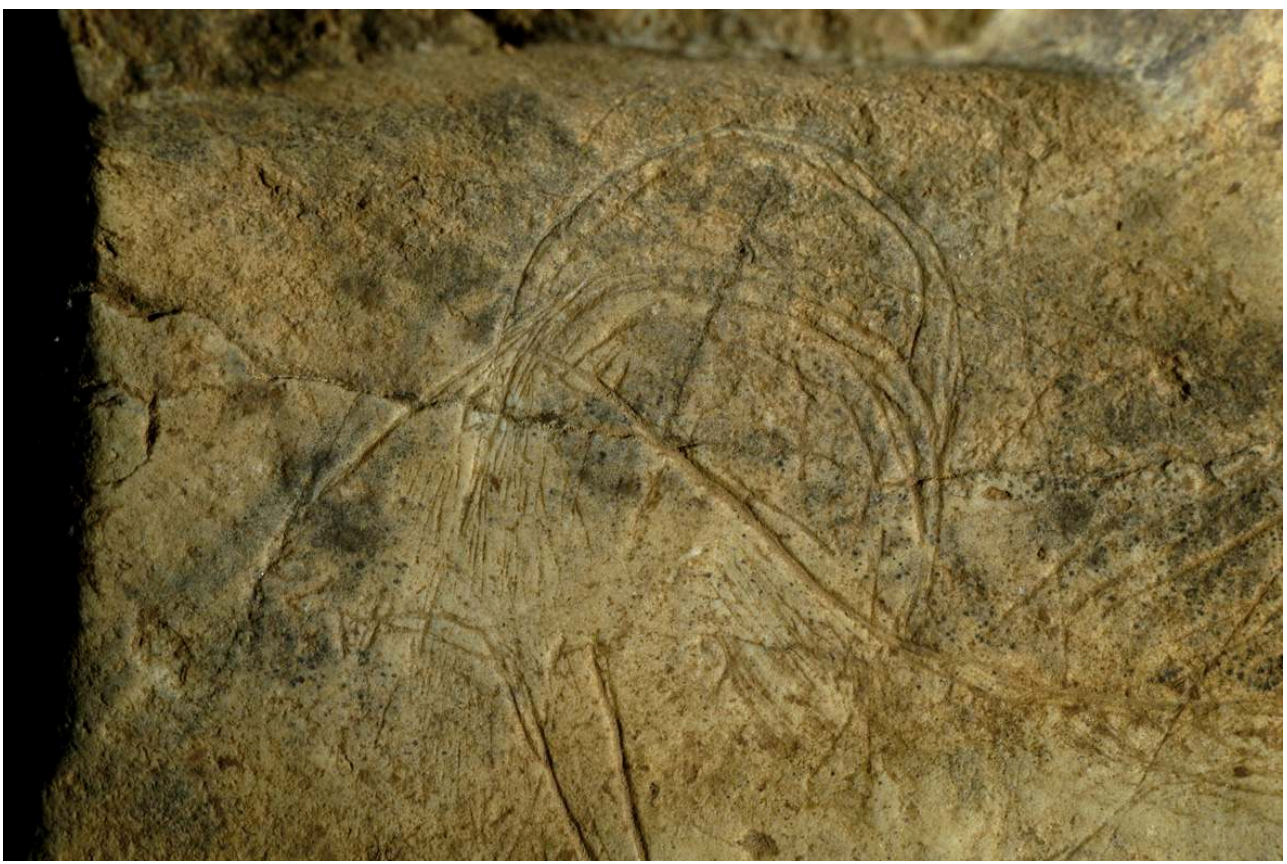

Tête du bouquetin dit « en marche », abri inférieur du Colombier

Phot. Marc Azéma. (C) Marc Azéma, avec l'autorisation du propriétaire, M. Henry Helly

La figure centrale du bloc représente un bouquetin mâle (fig. $\mathbf{n}^{\circ}$ 4), probablement le chef d'une harde (autres individus d'âges différents présents tout autour, notamment sous le ventre) trottinant vers la droite en bêlant. Cette image est exceptionnelle car elle constitue l'un des exemples les plus probants d'un processus de décomposition du mouvement par superposition d'images successives: les membres sont traités en contours multiples. Cinq à six contours ébauchés sont visibles entre les postérieurs écartés (fig. $\mathbf{n}^{\circ}$ 5), un ou deux en périphérie de l'antérieur droit. L'artiste magdalénien a manifestement cherché à traduire un flou dynamique afin de «donner vie » à cette figure. 
Figure 5

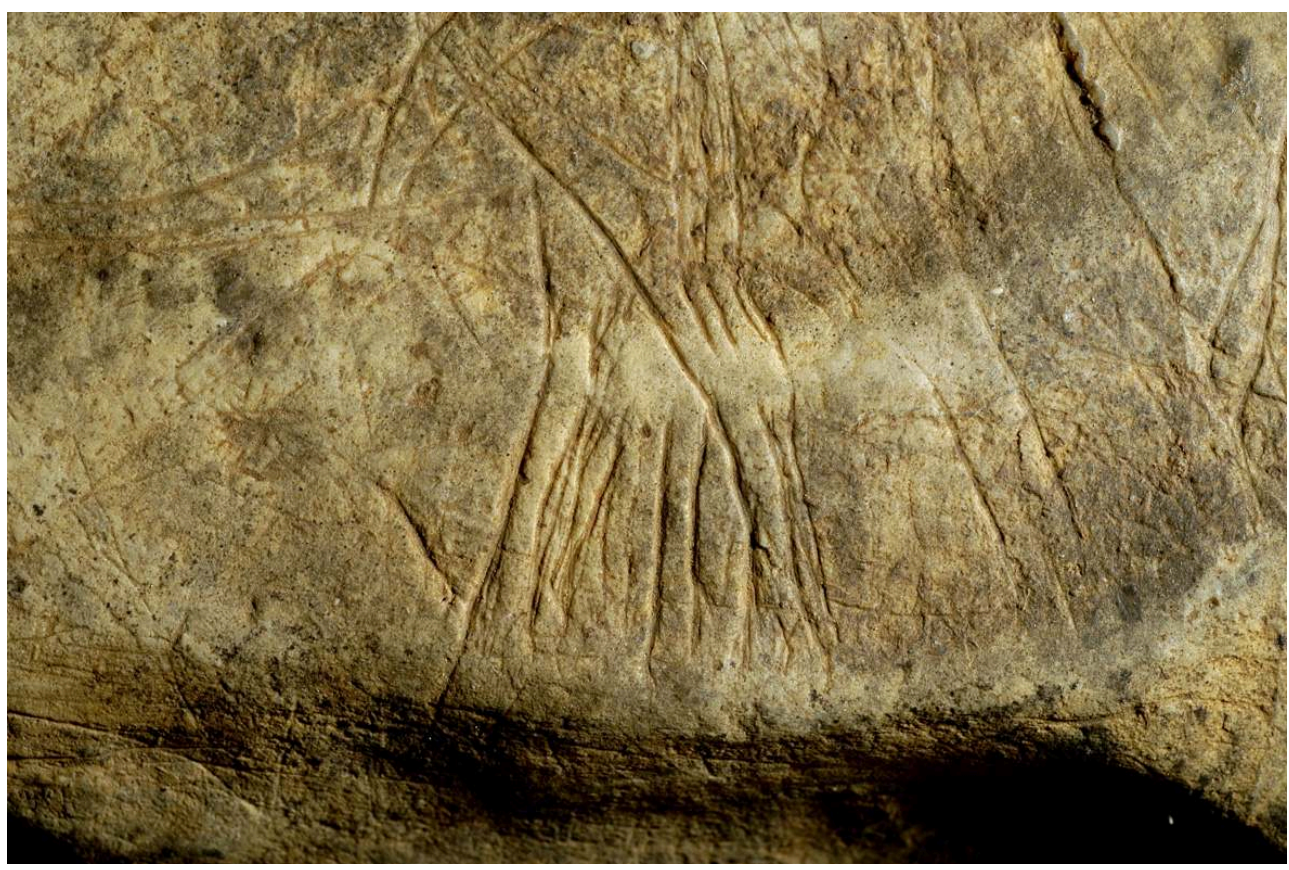

Détails des membres postérieurs du bouquetin dit « en marche » traités en contours multiples

Phot. Marc Azéma. (c) Marc Azéma, avec l'autorisation du propriétaire, M. Henry Helly régions de l'art paléolithique, tant dans l'art pariétal que l'art mobilier (voir notes 2, 5, $6,7,8,11,12$ ). Elle naît au Paléolithique dès l'Aurignacien comme en témoigne le bison à huit pattes du secteur des Chevaux de Chauvet ou le grand cervidé de la salle du fond de la même cavité, voisine du Colombier de quelques kilomètres seulement. Il atteint au Magdalénien, dans la même aire géographique, une perfection naturaliste avec ce bouquetin.

24 L'étude de cette figure va permettre pour la première fois de relever le plus précisément possible, sur la base de la numérisation 3D, l'ensemble des tracés laissés par l'outil de l'artiste sur le bloc. Grâce à la précision obtenue par le scan, cette opération expérimentale devrait permettre de mettre en évidence les gravures les plus fines, dont certaines, à l'état de "fantômes ", sont imperceptibles à l'œil nu et de les distinguer des artefacts de la roche: la manipulation sur station infographique de l'objet modélisé et texturé favorisera, par des variations d'échelle (zoom), d'angle et d'éclairage rasant, l'examen de la section des traits enregistrés, de les comparer afin de les caractériser (anthropiques ou naturels) et d'identifier le ou les outils à l'origine de leur élaboration (analyse technologique).

Ce travail de relevé (en cours de finalisation) doit aider à mettre en évidence la séquence d'élaboration de l'ensemble de la figure centrale et autoriser une approche fine du processus de décomposition du mouvement des membres. A l'échelle du bloc, il permet de mieux comprendre la mise en place des différentes unités graphiques (superposition) et de mieux appréhender la construction d'une composition à caractère naturaliste. 


\section{Déroulement de l'opération}

\section{Présentation de la méthode de relevé par numérisation 3D}

La lasergrammétrie est une science et une technique en plein essor qui exploite la technologie des scanners laser 3D (mesure laser haute densité, sans contact et longue portée). Un scanner 3D lève plusieurs millions de points en trois dimensions en quelques minutes. La mesure laser peut être couplée à une prise de vue photogrammétrique numérique. Avec cette technologie, le levé passe du domaine discret au domaine continu et de la 2D (ou 2.5D) à la vraie 3D. Développée à l'origine pour des applications industrielles, cette méthode de levé s'est étendue à presque tous les champs d'intervention du topographe.

Une intervention de numérisation en milieu souterrain pose des problèmes spécifiques liés aux points suivants :

\section{Difficultés logistiques}

Elles résident dans :

- le portage d'équipements lourds ;

- l'alimentation $220 \mathrm{~V}$ lorsque cela s'impose ;

- le monitoring des gaz ;

- la mise en sécurité des équipements et des hommes.

\section{Difficultés techniques}

Elles sont dues à :

- un milieu exigu (scanner au sol ou sur trépied court) ;

- une implantation topographique plus complexe et en particulier, la difficulté de rattachement au système de coordonnées de surface ;

- des stations de numérisation plus nombreuses du fait de la complexité géométrique du milieu ;

- un problème de manque de recul pour le scanner et pour les prises de vue ;

- une gestion des éclairages pour les prises de vue. 
Figure 6

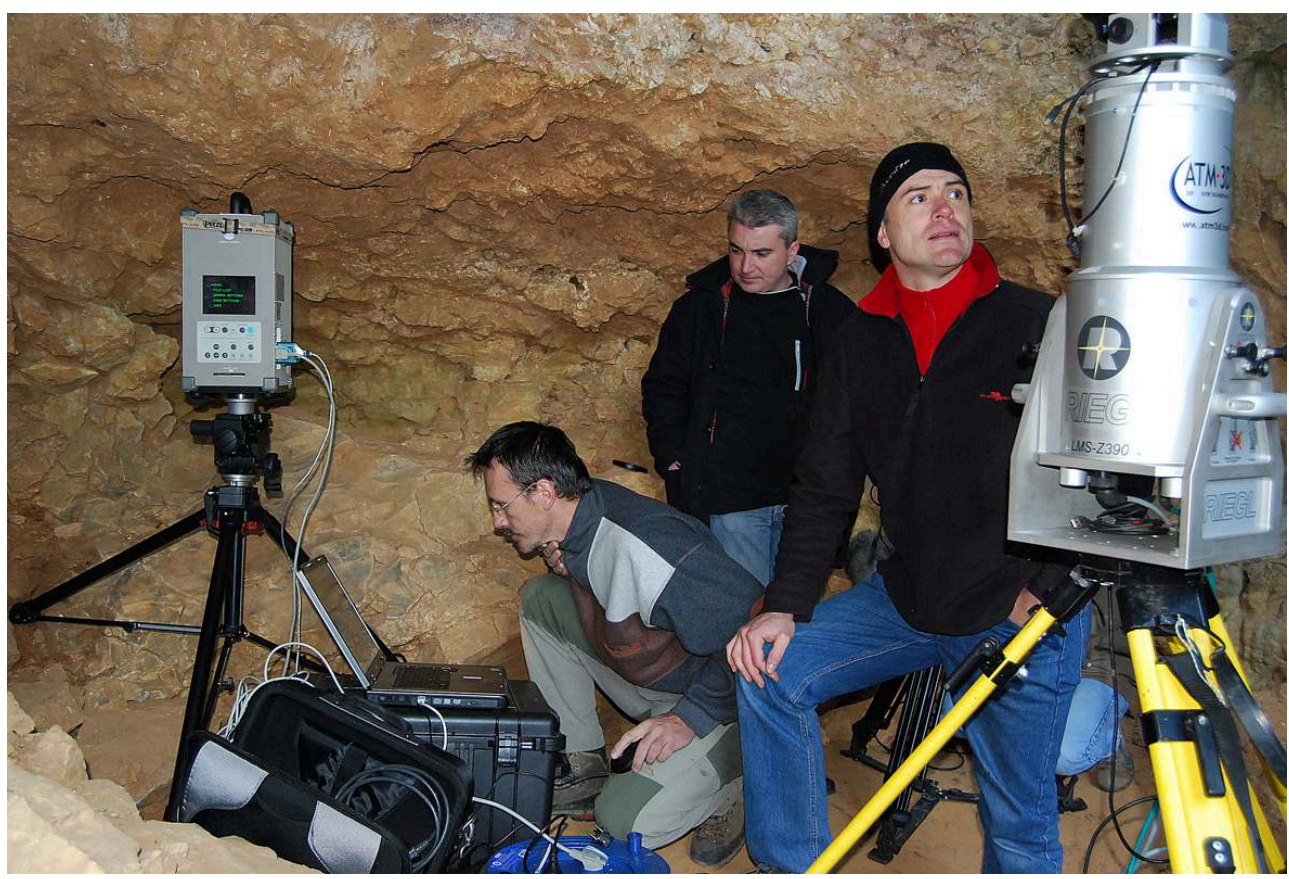

Mise en œuvre des deux scanners 3D dans l'abri inférieur du Colombier. Au premier plan : scanner longue portée destiné à la numérisation de l'ensemble de l'abri. Au second plan : scanner courte portée focalisé sur la paroi gravée

Phot. Françoise Prudhomme. (c) Musée de Préhistoire d'Orgnac, avec l'autorisation du propriétaire, M. Henry Helly

\section{Acquisition des données}

Dans le cadre de l'opération de l'abri du Colombier, l'acquisition des données a été effectuée par deux lasers scanners terrestres travaillant dans des gammes de précision et de portée différentes (fig. $\mathbf{n}^{\circ} \mathbf{6}$ ).

31 La numérisation 3D du bouquetin a été assurée par un scanner inframillimétrique Minolta VI 910 de précision $0,1 \mathrm{~mm}$. L'opération s'est déroulée en deux heures, auxquelles il convient d'ajouter le temps d'installation et de repli des équipements.

Les parois de l'ensemble de l'abri ont été numérisées par un scanner longue portée effectuant des mesures de distance à très haute fréquence $(10000 \mathrm{pts} / \mathrm{sec})$ dans toutes les directions de l'espace (fig. $\mathbf{n}^{\circ} \mathbf{7}$ ). Ce scanner produit un nuage de points en trois dimensions qui représente l'ensemble des impacts mesurés par le capteur. Le modèle mis en œuvre est un Riegl LMS Z90i de $400 \mathrm{~m}$ de portée et de précision $5 \mathrm{~mm}$. 


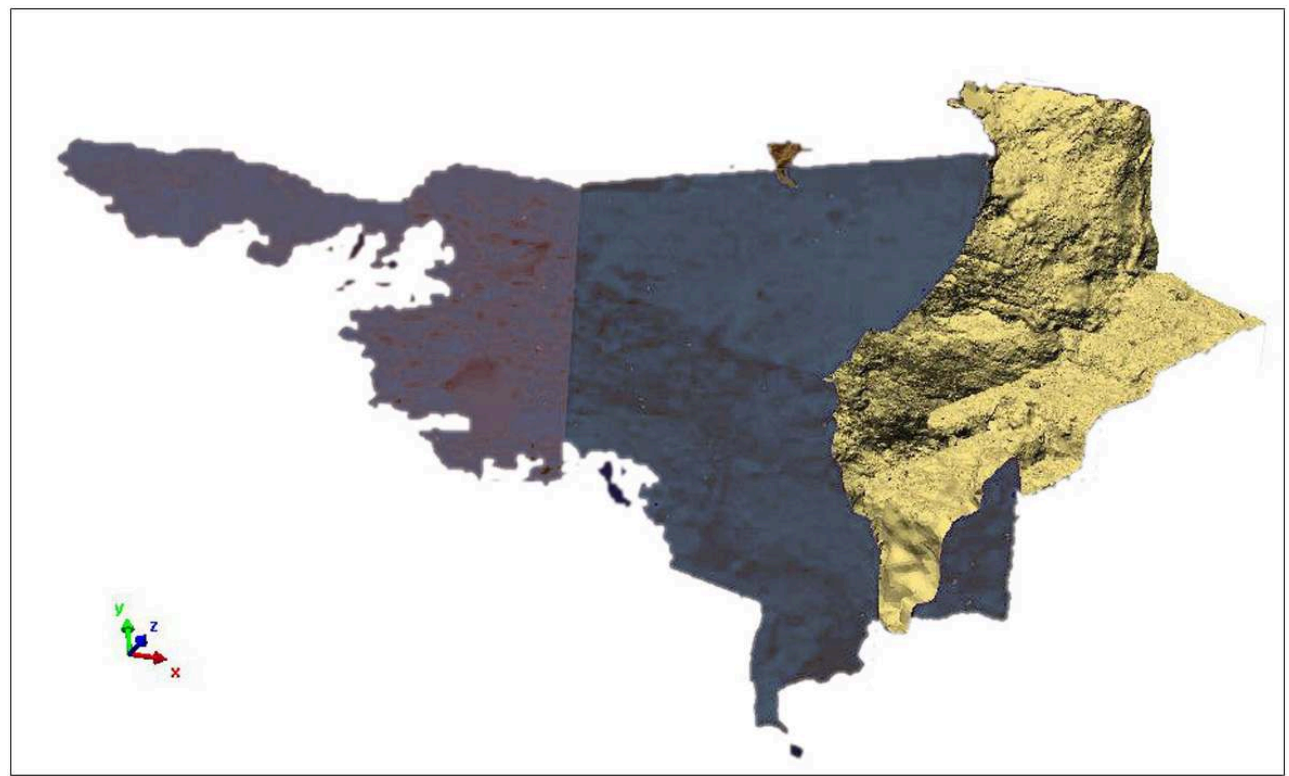

Vue générale du modèle numérique de l'abri inférieur du Colombier ; en jaune l'ouverture

Document ATM 3D (c) Musée de Préhistoire d'Orgnac, avec l'autorisation du propriétaire, M. Henry Helly

Le levé au scanner à partir de plusieurs stations produit un nuage de points géoréférencés. Chaque point est connu en planimétrie, altimétrie, intensité de réflexion du laser et éventuellement colorimétrie. Un projet produit généralement au minimum quelques millions de points.

\section{Résultats}

Quel que soit le type de capteur utilisé, le résultat d'une opération de numérisation est avant tout un fichier numérique contenant une série de coordonnées géométriques $(\mathrm{X}, \mathrm{Y}, \mathrm{Z})$, constituant ce qu'on appelle un nuage de points 3D. Au nuage de points sont parfois associées la couleur du point (RVB), l'intensité de réflexion et les prises de vues numériques calibrées et géoréférencées. Ces données, issues d'une campagne de levé, sont alors les informations de base, vierges de tout traitement, pouvant constituer un document brut de l'objet, aux critères de résolution et d'accessibilité visuelle près.

Le résultat est un semis de points propre dont la densité et la continuité dépendent des conditions de terrain et de la densité choisie pendant l'acquisition. C'est ce résultat qui sera exploité pour la modélisation de l'objet. 
Figure 8

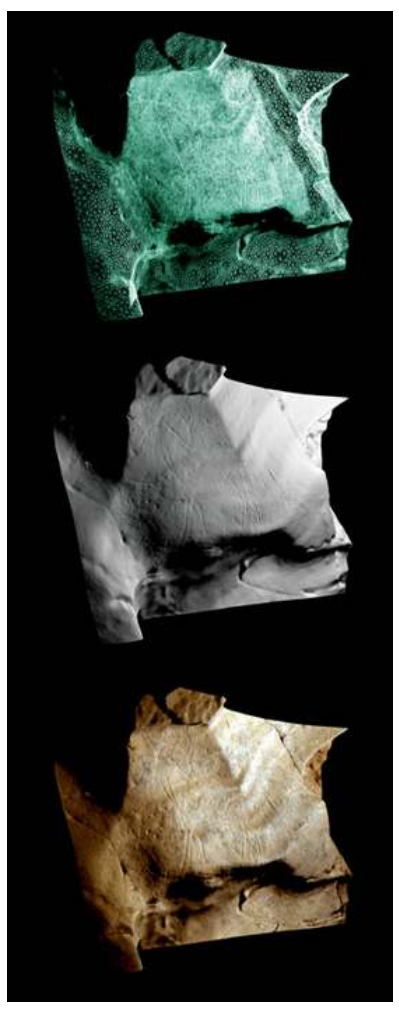

Le panneau du bouquetin de l'abri inférieur du Colombier. Haut : modèle triangulé avec deux niveaux de précision $(0,1 \mathrm{~mm}$ pour la gravure du bouquetin à pattes multiples). Milieu : modèle surfacé. Bas : modèle texturé tel qu'il apparaît dans l'animation visible dans l'exposition organisée par le musée régional de Préhistoire d'Orgnac et le Centre de Préhistoire de Pech Merle à Cabrerets

Phot. ATM 3D / Passé Simple @ C Musée de Préhistoire d'Orgnac, avec l'autorisation du propriétaire, M. Henry Helly

Un modèle maillé tridimensionnel est une véritable peau numérique qui permet de reconstituer la géométrie de la surface numérisée. La densité des triangles est soit équivalente à celle des points acquis soit simplifiée en fonction de critères géométriques précis compatibles avec les caractéristiques de l'objet (fig. $\mathbf{n}^{\circ} \mathbf{8}$ ) (haut).

Une fois le modèle surfacique réalisé et optimisé (voir fig. $\mathbf{n}^{\circ} \mathbf{8}$, milieu), on procède à la «colorisation" du modèle 3D (voir fig. $\mathbf{n}^{\circ} \mathbf{8}$, bas). Les prises de vues photographiques utilisées pour cette opération sont issues d'une optique et d'un appareil photographique calibrés permettant de réaliser des clichés de très haute définition (17 millions de pixels).

Par le calcul, on vient replacer chaque pixel de la photographie (2D) dans le modèle 3D. Le texturage précis du modèle triangulé va contribuer à augmenter l'information disponible. Certains reliefs trop fins (traits de barbiche ou pelage de la joue du bouquetin) n'ont pas été enregistrés par le scanner mais par la photographie.

\section{Perspectives}

La mise au point de l'application de cette technique aux relevés de gravures pariétales fines peut permettre de travailler, dans un second temps, sur les autres vestiges pariétaux de l'abri et de la grotte adjacente et, à terme, dans deux autres cavités du secteur : la grotte des Deux Ouvertures et la grotte d'Oulen où les conditions d'accès, de 
travail et de lisibilité des œuvres sont particulièrement délicates. Plus globalement, cette technique devrait être mise en œuvre sur l'ensemble des cavités ornées paléolithiques et holocènes du secteur. L'application de ce procédé permettra un meilleur enregistrement (archivage) et un véritable suivi objectif de l'état de conservation des œuvres et du support rocheux.

Figure 9

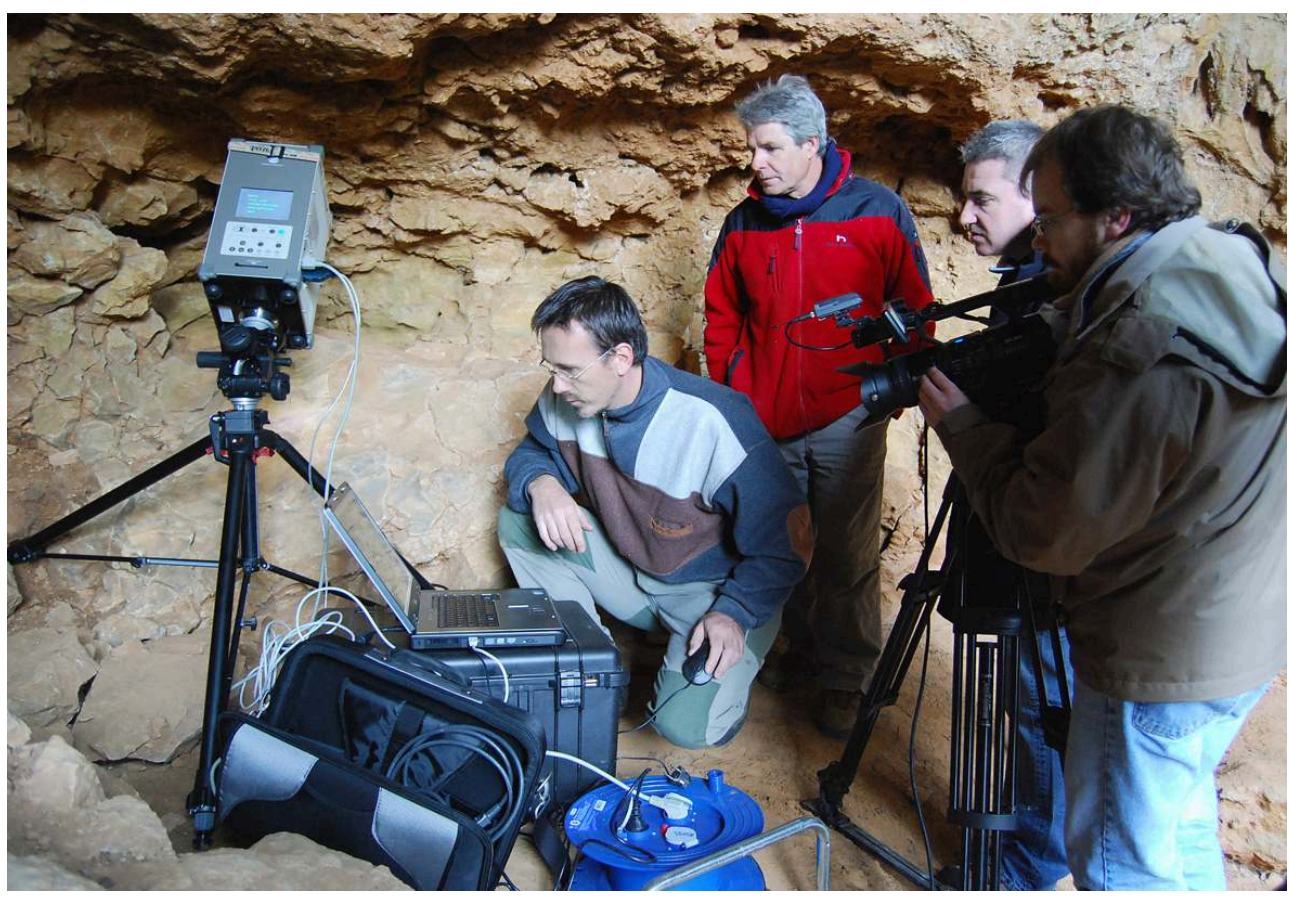

L'opération de scan du panneau gravé de l'abri du Colombier a fait l'objet d'un archivage vidéo HD par David Geoffroy et Passé Simple

Phot. Françoise Prud'homme. (c) Musée de Préhistoire d'Orgnac, avec l'autorisation du propriétaire, M. Henry Helly

Dans le cas précis de l'abri du Colombier, à moyen terme, nous envisageons d'étendre la numérisation inframillimétrique aux autres lambeaux de parois ornées encore en place, à ceux détachés et enfouis dans le sol archéologique, retrouvés lors des fouilles Onoratini et d'autres éventuellement dégagés lors d'une fouille future. Une fois cette opération conduite, une restauration virtuelle de la paroi pourrait être envisagée, à l'image de celle déjà réalisée pour la grotte de Marsoulas en Haute-Garonne ${ }^{17}{ }^{18}$ ou à venir dans la grotte gardoise de Baume Latrone (étude en cours dirigée par Marc Azéma).

41 Signalons enfin que l'opération de scan 3D du Colombier a fait l'objet d'un archivage vidéo (réalisation Passé Simple et David Geoffroy) destiné à produire un vidéogramme pédagogique visible dans l'exposition « Préhistoire de la bande dessinée et du dessin animé » (fig. $\mathbf{n}^{\circ}$ 9). 


\section{in Institut national du patrimoine}

Communication présentée lors du séminaire ART RUPESTRE : LA 3D UN OUTIL DE MÉDIATION DU RÉEL INVISIBLE ? qui s'est tenu du 4 au 6 juin 2008 à Angles-sur-l'Anglin, coordonné par l'Institut national du patrimoine, avec la participation de la direction de l'architecture et du patrimoine

\section{NOTES}

1. Exposition temporaire: avril-novembre 2008 au musée régional de Préhistoire d'Orgnac (Ardèche) ; avril-novembre 2009 au Centre de préhistoire du Pech-Merle de Cabrerets (Lot).

2. Azéma, Marc. Préhistoire de la bande dessinée et du dessin-animé. Catalogue d'exposition, 2008, musée de Préhistoire d'Orgnac - Centre du Pech-Merle - Passé Simple.

3. Voir le site www.orgnac.com.

4. Passé Simple, Société de production d'expositions et de films documentaires sur le patrimoine, l'archéologie, les arts : www.passesimple.net.

5. Azéma, Marc. La représentation du mouvement dans l'art pariétal français. Approche éthologique du bestiaire, Thèse de Doctorat. Marseille : Université de Provence, Aix-Marseille I, 2003, 3 vol.

6. Azéma, Marc. Les origines préhistoriques de la bande dessinée et du dessin animé, Science et Vie, 2005.

7. Azéma, Marc. La représentation du mouvement au Paléolithique supérieur. Apport du comparatisme ethnographique à l'interprétation de l'art pariétal, Bulletin de la Société Préhistorique Française, 2006, t. 103, n 3, p. 479-505.

8. Azéma, Marc. Representation of movement in Palaeolithic parietal art. An ethnographical approach, Anthropozoologica, 2008, tome 43, fasc. 1, p. 117-154 (DVD).

9. Azéma, Marc. La représentation du mouvement dans l'art animalier paléolithique des Pyrénées. Bulletin de la Société Préhistorique Ariège-Pyrénées, 1992, tome XLVII, p. 19-76.

10. Azéma, Marc. La décomposition du mouvement dans l'art animalier paléolithique des Pyrénées. Préhistoire et Anthropologie Méditerranéenne, 1992, tome 1, p. 17-31.

11. Azéma, Marc. La décomposition du mouvement dans l'art paléolithique. International Newsletter on Rock Art, 2005, $n^{\circ}$ 43, p. 14-21.

12. Azéma, Marc. Et si... les hommes préhistoriques avaient inventé le dessin animé et la bande dessinée ? Préhistoire, Art et Sociétés, 2005, tome LIX, p. 55-69.

13. Combier, Jean, Porte, Jean-Louis, Ayroles, Pierre, Gély, Bernard. Abri du Colombier. In : LeroiGourhan A. (dir.). L'art des Cavernes, 1984. Paris: Ministère de la Culture - Imprimerie nationale, p. 621-625.

14. Combier, Jean. Le Paléolithique de l'Ardèche dans son cadre paléoclimatique. Mémoire de l'Institut de Préhistoire de l'Université de Bordeaux, nº 4. Bordeaux : Éditions Delmas, 1967, p. 342 . 
15. Onoratini, Gérard, Combier, Jean, Ayroles, Pierre. Datation $14 \mathrm{C}$ d'une gravure pariétale de bouquetin de l'abri magdalénien du Colombier à Vallon-Pont-d'Arc. C.R. de l'Académie des Sciences de Paris, tome 314, série II, 1992, p. 405-410.

16. Autorisations préalables à l'enregistrement : le propriétaire de la cavité, le service régional de l'archéologie et la Réserve nationale naturelle des gorges de l'Ardèche ont adhéré au projet et fourni les autorisations nécessaires. Le SRA et le gestionnaire de la réserve ont mis des agents à disposition pour le suivi de l'opération.

17. Fritz, Carole, Azéma, Marc, Tosello, Gilles, Moreau, Olivier. La restauration virtuelle de l'art des cavernes de la Préhistoire. Archéologia, 2009, nº 469.

18. Voir, dans ce numéro, les articles de : Fritz, Carole, Tosello, Gilles, Azéma, Marc, Moreau, Olivier, Perazio Guy et Péral, José. Restauration virtuelle de l'art pariétal paléolithique : le cas de la grotte de Marsoulas. [Document électronique]. Paris: Ministère de la Culture et de la Communication. Fritz, Carole, Tosello, Gilles, Perazio Guy, Péral, José, Guichard, Lionel. Technologie 3D et relevé d'art pariétal: une application inédite dans la grotte de Marsoulas. [Document électronique]. Paris : Ministère de la Culture et de la Communication.

\section{AUTEURS}

\section{MARC AZÉMA}

Docteur en Préhistoire, chercheur associé à l'Unité Mixte de Recherche 5608 du CNRS (Toulouse Le Mirail/UTAH) et au Centre Cartailhac pour la Recherche et l'Étude de l'Art Préhistorique (CREAP) ; réalisateur de documentaires. marc.azema@wanadoo.fr

\section{BERNARD GÉLY}

Préhistorien, Service régional de l'archéologie, Direction régionale des affaires culturelles de Rhône-Alpes. bernard.gely@culture.gouv.fr

\section{FRANÇOISE PRUDHOMME}

Responsable du Musée régional de Préhistoire d'Orgnac. f.prudhomme@orgnac.com

\section{ATM 3D SOCIÉTÉ ATM3D}

spécialisée dans la mesure par scanner laser 3D, discipline appelée lasergrammétrie ou lidargrammétrie.contact@atm3d.com 